\title{
Service Composition in Public Networks: Results from a quasi-experiment
}

\author{
Ralph Feenstra \\ Faculty of Technology, Policy and Management, \\ Delft University of Technology, \\ Jaffalaan 5, NL-2600 GA, Delft, The Netherlands, \\ r.w.feenstra@linqworks.com
}

\author{
Marijn Janssen \\ Faculty of Technology, Policy and Management, \\ Delft University of Technology, \\ Jaffalaan 5, NL-2600 GA, Delft, The Netherlands, \\ m.f.w.h.a.janssen@tudelft.nl
}

\begin{abstract}
New systems can be created by assembling a set of elementary services provided by various stakeholders in a service composition. Service composition is not a trivial endeavor and several composition methods exist. Yet, these methods are hardly adopted by the field, as they need a set of well-defined and welldescribed services and are not able to deal with incomplete information and unambiguous descriptions. Furthermore, these methods are not able to deal with situations in which stakeholders have different requirements and need to negotiate with each other about the selection and performance of services.

In this paper we present a service composition method and evaluate this method using a quasiexperimental design. The method is aimed at dealing with a set of heterogeneous services provided by stakeholders with different and sometimes even opposing requirements. We found that our composition method helps to create an overview of services and their attributes and enables better communication between stakeholders. A recommendation for further research is to integrate semantic and multi-actor composition methods.
\end{abstract}

\section{Introduction}

Organizations are more and more forced to collaborate with each other, share information and reuse functionality to reduce cost. This duplication of efforts is founded in the highly fragmented and unrelated applications in which each organization has developed applications independently of other organizations. Many of these applications overlap in functionality and content and are in fact 'isolated islands of information and/or functionality' [1].

At present, there is a trend towards creating new systems by composing them out of single web services, known as web services compositions or compositions for short [2]. Web services are selfcontained, self-describing, software modules that can be published, and remotely invoked and in this way reused [3]. A composition combines a set of web services following a certain composition pattern to achieve a certain objective [4]. Such compositions are synthesized by the reuse of elementary web services. New systems can be created by reusing existing software components and in this way avoiding a large duplication of efforts. Yet the benefits of reusing information and functionality have not been attained, as service composition is a difficult endeavor [5].

Many governmental organizations have already developed software that can be accessed as web services. By invoking these web services the functionality can be reused or information can be accessed. There are varying motives for choosing the modularization strategy. Sharing services can provide efficiency gains, access to services that are otherwise out-of-reach given the limited resources, and can also fasten the development process [6]. Furthermore, several services provided by multiple government agencies, in this way acting as service providers, need to be combined, e.g. composed, to create a new service. An example of a simple composition is the online checking of a car by a police officer on the street using a mobile device. The composite service consists of the five elementary services: 1) checking the license plate and owner information at the RDW (the Dutch road traffic department), 2) checking if the taxes have been paid at the Inland Revenue Service, 3) checking if all fines have been paid in the police databases 4) validating the name and address information of the driver in a municipal database, each municipality has its own database containing information about citizens within their geographical boundaries, and 5) merging the results of the four previously mentioned web services on a single page.

The creation of a composition within a public service network can easily be viewed as a simple and straightforward process, but in fact is a very complex endeavor [5]. What might be viewed as a process of selecting component can easily result in the need to 
develop new components, struggles about the interpretation of functionality and performance and finally result in expensive and time-consuming implementation projects. Existing composition methods assume the presence of a set of interoperable services, which is often not a realistic assumption [7]. Further, Liang, Huang and Chuang found that there were no publications that take into account the constraints of non-functional service properties [2] and Feenstra, Janssen and Wagenaar [5] found that constrains and problems originating from multiple stakeholders situations are ignored. The use of existing services can complicate the reuse process, as the modules are not determined using a conscious design process and the effectiveness of a "modularization" is dependent upon the criteria used in dividing the system into modules [8]. As a result, existing composition methods are not adopted by the field.

The goal of this paper is to evaluate the merits and disadvantage of the multi-actor composition method derived in previous research [9] in comparison to other methods. In the following section we discuss the theoretical background followed by our research approach. In section four the composition method is presented and subsequently evaluated in section five. Finally, we discuss the results and draw conclusions.

\section{Background}

\subsection{Public service networks}

Public organizations have to collaborate in networks to benefit from each other's resources. The need for cooperation and sense of urgency in these networks will become stronger as society expects better performance [10]. These public networks consist of many organizations who can act autonomously. Designing a service composition in a network of agencies involves addressing many challenges outside the control of a single agency [11]. Such organizational networks can be viewed as complex systems consisting of a large number of independent entities that seek to accomplish their own goals, but operate according to some rules and norms in the context of relationship with the other entities. An organization can be viewed as "a set of multiple, goalsshifting political coalitions" [12]. The actors have mostly different interests and are dependent of each other. Due to these dependencies no single actor can solve the problem autonomously. Further, none of the actors can impose a particular solution on the others. In public networks, the creation of solutions depends on mutual agreement and understanding [10]. The creation of solution has to take into account contradictory goals and interests. This is an important starting point in public sector engineering projects, as no actor can tell others what to do or what to think. Consequently it is important that all actors involved are able to express their own point of view and no solution is chosen in advance.

The decision makers involved in designing compositions face a difficult task because of the large set of alternatives and the difficulty to estimate the effects of a composition design on all parts of the organization. Human decision making capacity is limited, persons can only deal with a limited set of details [13], in this case a limited set of alternative compositions. The limits to human information handling capacity introduce a non rational element to the decision making. This element grows larger if the decision makers are overwhelmed with detailed information regarding. The limits to human rationality are often described using the term bounded rationality [13].

Often no single 'best solution' exists. Public networks are characterized by interdependence between actors, differences between actors that interfere with cooperation, and reticence of actors that don't see their interests met by choosing a particular solution and dynamics regarding the group of actors involved in the decision making process [14]. As a result, the problem addressed cannot be seen as a 'hard science' multidimensional optimization process that can be solved using mathematic algorithms. One stakeholder might favor efficiency over service levels, whereas another stakeholder might have opposing preferences still the solution should satisfy both stakeholders. It is impossible to derive a formal specification of the preferences of each actor in every situation, as these might not be necessarily based on rational reasons.

The actors in the network will need to cooperate to a certain extent in order to realize a solution that at the same time helps realizing common goal and pays sufficient attention to their specific interests. The decision making process is effective only if it leads to commonly supported decisions [15]. The realization and success of compositions in a multi-actor environment strongly depends on the participation of the players involved. Collaboration is complicated as different stakeholders will be used to different names and terms (syntax and semantics) to describe services. This all results in the need for a design process of service composition in which stakeholders actively participate. Consequently, the design process that leads to a service composition should result in a commonly accepted decision. 


\subsection{Existing service composition methods}

Service composition methods are dominated by rational approaches in which a system consist of parts. Functional decomposition is a common approach found in systems theory [16]. The idea is that a system can be decomposed into parts, each part can be implemented and in turn integrated. Functional decomposition refers broadly to the process of resolving a functional relationship into its constituent parts in such a way that the original function can be reconstructed (i.e., recomposed) from those parts by composition. This assumes that an analysis is made first and then the functional components are determined. In the situation of designing a composition many organizations already have developed services and there is no greenfield situation. Instead a compositions needs to be created from services that might not be designed for that purpose.

Several evaluations of the compositions approaches can be found in literature [2, 5, 7, 17]. Milanovic and Malek [7] and Beek [17] compare composition approaches. Milanovic and Malek using the following criteria 1. Service connectivity, 2. Non-functional properties, 3. Composition correctness, 4. Automatic composition, and 5. Composition scalability. Beek et al. [17] extended the set of criteria used by Milanovic and Malek by adding 6) exception handling and compensations and by including 7) tool support. The composition approaches are inspired by the idea that there is a repository that includes a set of well-defined and standardized services that can be used to create a new system. They found that all composition approaches offer connectivity. Without connectivity if would be impossible to integrate a set of services. Connectivity is an absolute minimal requirement for a composition approach. Yet, modifications that might be needed to create interoperability are not part of these methods. They rather assume that a set of interoperable services exists. This is not a realistic starting point as in public networks services are often not developed for the purpose of reuse and are not interoperable.

Already existing services can complicate the reuse process, as the modules are not determined using a conscious design process. In addition composition methods expect a well-defined and interoperable set of services as input [7]. Often services are developed without having reuse in mind. According to Parnas [8] the effectiveness of a "modularization" is dependent upon the criteria used in dividing the system into modules.

Milanovi and Malek [7] and Beek [17] found that most composition approaches neglect specification of non-functional properties. Only OWL-S lets users define non-functional properties, but that capability has yet to be fully specified [7]. Liang, Huang and Chuang found that there were no publications that take into account the constraints of non-functional service properties [2]. These kinds of properties include cost, security, dependability, response time, reliability and scalability. In decision-making concerning the selection of services these non-functional properties have a large influence. A typical concern of stakeholders has to do with a trade-off in nonfunctional requirements and as such it is essential that they are addressed.

The analysis and selection of services for Web services composition is, in general, much more complex than the analysis and selection of parts for product design because selection criteria are difficult to define and composition components are diverse [2]. Different stakeholders might have different selection criteria and look at other types of characteristics. Components can be small or large grained, might be configurable and interfaces might be completely different. In conclusion, there is a need for a composition method that is able to deal with a variety of stakeholders having different interests while providing all kind of elementary services that need to be combined in a service composition. In the following subsection we will derive requirements on such a method.

\subsection{Requirements on a service composition method for public networks}

Existing composition methods assume that all services are well-defined and that there are unambiguous selection requirements and that there is a clear, undisputed goal regarding the performance. In fact, these approaches can be better denoted using the term representational formalism than using the term composition approach [5]. The existing methods lack some crucial aspects which are essential for the successful creation of service compositions in public networks. Based on the confrontation of current service composition methods and the characteristics of public networks, the following problems and requirements on a service composition method for public networks can be derived:

1. Dealing with incomplete information. Stakeholders are not able to provide, or might even not want to provide, an overview of all their services in advance. Some information might be available whereas other information might be omitted;

2. Demand-driven: Current composition methods use 
existing services and in this way take the supply as a starting point. Not the problem but the solution is central in these methods. Instead, the need to solve a certain problem should be the starting point and the service composition solution should solve this need;

3. Dealing with non-existing services: Some services exist, others are under development or the development has been planned for. Furthermore, management might wonder which services need to be developed in the future. Existing methods cannot support the planning process;

4. Including non-functional requirements: Most compositions methods neglect non-functional requirements and only some methods use quality metrics [2]. In many cases no hard data about quality metrics are available. This tacit knowledge need to be mobilized;

5. Considering various stakeholder views: There might be different stakeholder requirements and different stakeholders might prefer different solutions. For example, one party might focus on the cost, whereas others might be more interested in reliability. There is a need for negotiation and supporting the trade-off during a service composition process.

6. Dealing with ambiguous interpretation: Requirements, functionality and performance might be interpreted differently by stakeholders. Therefore the creation of a shared view is crucial. For example, identity management might be interpreted as a service used for acquiring basic data about persons or as a facility that can support authentication. Both interpretations might be correct, but might also be incorrect in a specific case. Various actors might give different interpretations to the functionality description.

These requirements will serve as a starting point for the development of the composition method described in this paper. In the following section we will discuss the research approach to develop and test a composition method that is better equipped to deal with the specific aspects characterizing public networks.

\section{Research design: quasi-experiment}

A composition method is necessary to overcome the identified hurdles. In design research, a method is often developed by elicitating a number of requirements that can help to overcome the problems that resulted in the need for the method. Next, the method is evaluated using the requirements and the ability to overcome the problems. A method is thus usually evaluated using the requirements posed on a method. Instead of only evaluating the method based on the requirements, we opted for quasiexperimentation in which our method is evaluated for the ability to solve the identified problem and at the same time compared with another composition method and its ability to deal with the problems. This approach has the advantage that more insight into the potential and limitations of this design method can be gained. A quasi-experiment is a study in which interventions, in our case two compositions methods, are deliberately introduced to observe their effects, by non-randomly assigning the units [18]. To assess the impact of the newly designed multi-actor composition method, different methods are used to solve exactly the same case. In this way the conditions remain the same and the difference in outcomes are contributed to the different composition methods.

A workshop approach was chosen because it enables the comparison of different composition approaches within a controlled environment and did not require long term efforts from busy professionals. During the workshops, the participants designed compositions for several given scenarios within a relatively short time. In total, there were 21 participants during a 4-hour workshop. All participants were experienced in the field and were able to compare our composition method with their own practices.

Each of the scenarios used to evaluate the method consisted of an objective, a description of the composition that has to be realized, an overview of the available services and an overview of the actor specific demands. The scenario and roles were derived from a real-life example to ensure that the scenario resembled real life as close as possible. The scenario dealt with the construction of a new service in which a variety of stakeholders had to cooperate and make use of each other's services.

The workshop resembled the real situation closely, as multiple parties (actors) having various objectives had to design one composition. This means that they had to search for possible solutions, negotiated on the use of certain services, and the development of new services. The workshop required only a short amount of time and offered the possibility to vary both the composition method used and the available ICT support (the software) for the method.

Figure 1 depicts the structure of the quasiexperiment. Two types of workshops were held, using exactly the same scenario as input to ensure that the methods are evaluated using the same circumstances. In one workshop the participants could use their own method as used by their organizations. We label their own method as best practice method, as they are using 
the methods used by their own organization which is a based on aspects like their organizational standards and past experiences. In the other workshop the composition method presented in the following section was used by the participants. Comparison of these methods is possible as both have public networks as a starting point. We did not choose to compare our method with existing composition methods, as they are based on other starting points which do not match the scenarios which are derived from practice.

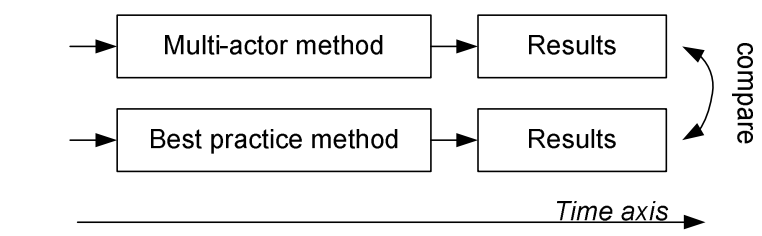

\section{Figure 1: The composition evaluation approach}

At the end of both workshops, the opinions of the participants regarding the method and the provided support were asked. For this purpose, a survey was developed, based on the problems and requirements discussed in section two, aimed at evaluating the main characteristics of our composition method. The results of the survey were analyzed using SPSS. Next to the quantitative comparison of the method, the behavior of the workshop participants was observed and interviews were held. This resulted in a qualitative evaluation of the strengths and weaknesses of the best practice and multi-actor method.

\section{Multi-actor service composition method}

System development is often viewed as a highly complex process which is difficult to structure and has many interactions. In such situations a variety of different mental templates or cognitive filters can be used to influence managerial decision making [19]. Methods can be used to guide actions and decisions. The aim of this composition method is to improve the development process. This should help to simplify complicated composition processes and provide knowledge at an appropriate level of abstraction for decision-makers. We opted for an approach consisting of steps and support for each of these steps, aimed at stimulating both the interactions among participants and the group processes. The basic idea is that in this way the majority of stakeholders are represented and tactical (expert) knowledge is mobilized. The main differences with existing composition methods are that we take the involvement of multiple actors in a public network as a starting point instead of a given set of services. Therefore the method is named Multi-Actor Service Composition method. The service composition method consists of a number of steps and support for these steps that will be briefly discussed in the following subsections, an extensive descriptions can be found in $[5,9]$.

\subsection{Service composition steps}

Our method is based on the idea that services are not well-described, and if they are described the description might be ambiguous. The need (demand) to solve a certain problem is the starting point instead of the supply of services. Therefore the involvement of stakeholders is necessary to 1) represent their goals and purposes and 2) to mobilize their knowledge. The system development process is viewed as an interactive process where many parties with different interests can negotiate on the outcomes. Nevertheless, this process needs to be guided. Burstein [20] used three main steps service discovery, engagement and enactment. Our efforts are concentrated on service discovery and engagement and not on enactment, as new services might need to be modified or developed before execution is possible. Our method is based on the requirements on the compositions as a starting point and therefore a requirement specification step is added. In addition, we wanted to have the evaluation of possible compositions as an explicit step, which might result in increasing understanding and the identification of new compositions. As a result, our method consists of five steps, depicted in Figure 2, that are always part of a service composition development process. These steps provide guidance at a high level of abstraction and could help decision-makers to deal with a conscious development process.

The steps are interactive and there is no pre-defined sequence. The only condition is that all step need to be completed before a service composition is created.

\section{Requirements specification}

The first stage focuses on identifying and understanding the need for a new system or service. This stage starts with rough ideas concerning the desired functionality and finishes with a structured list of desired properties of the design.

\section{Description of existing services}

Stakeholders need to communicate with each other and discuss the use of possible services. For this purpose, a service register is created in collaboration with the stakeholders. Not all data need to be included in the register, as they might not be available. Moreover, services that are under development can be included. Decisions on the attributes that will be included in the 
register have to be made. Probably someone is held responsible for the correctness of the descriptions. The maintaining of this service register can be a continuous effort.

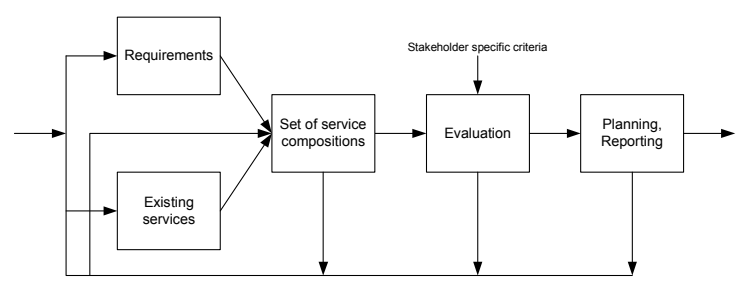

Figure 2: The five essential steps of the service composition process

3. Design of sets of service compositions

In this phase the participants discuss and negotiate possible service compositions. The compositions are designed and each stakeholder might design his or her own composition.

4. Evaluation of the composition designs

The designs created in the previous step need to be assessed. Evaluation can be used to select a single composition or a subset of compositions for implementation or for further investigation and refinement. Various evaluation methods can be supported and evaluations might be from various stakeholder perspectives.

5. Planning and reporting

During the last step the decisions that were made are listed including their motivation. Furthermore, a list of changes to existing services that are needed and/or a list of new (to be developed) services is made.

A starting point of our method is that all actors are able to express their own point of view and no solution direction is chosen in advance. Consequently, these steps should stimulate dialogues among stakeholders and clarify the assumptions on underlying views of other stakeholders. In this way judgmental pitfalls and advocacy of ones own viewpoint without support by any facts or explicit mentioning of assumptions can be avoided. For example, the steps should help to make explicit that a participant assumes that a service is reliable while there is no information to support this. This might result in the need for more research or the creation of a set of shared starting points that are plausible. The focus of the method is on exploiting rather than eliminating each others' viewpoints. Schein [21] argues that experiencing and acknowledging differences of interpretations is a critical step for further inquiry of underlying assumptions. These rules of the composition design process will be communicated in advance.
The steps described above need to be taken, but our method is not aimed at prescribing the sequence of steps or iterations. Our starting point is the knowledge in the organization that needs to be used in the process and consequently stakeholder interaction and exchange of knowledge to come up with better and more feasible service compositions is key. In the next subsection we describe the support for these steps.

\subsection{Service composition support}

The service composition steps indicate the essential steps that need to be taken. A prototype was developed that support each of these steps. The central idea of the support for these steps is the use of the service portfolios concept, analogous to the concept of application portfolios [e.g. 22]. The service portfolio is intended to support decision makers involved in a service composition process. The prototype consists of the following elements.

1. A centralized database containing all existing services described using codified service data. This database contains both functional and nonfunctional properties of services. Stakeholders can add properties and in this way include their own evaluation criteria. For example, if a stakeholder thinks it is necessary, the stakeholder can add reliability as a non-functional property.

2. The database includes current services and services that will come available in short time and identifies services that need to be developed in order to support new developments;

3. The service portfolio describes the desired composite service that needs to be developed. In this say not only the supply of elementary services is described, but also the needs and requirements on the new services from the various stakeholders' perspectives is included.

The service portfolio can be extended during the service composition process and stakeholders can express their own view on the desired composition.

Various commenting, sorting and ranking methods were included, as actors might favor their own way of evaluation or might have different standards for evaluation. Aspects like costs, reliability, continuity, availability, reliability and performance of services might be decisive factors and should be included in the evaluation of compositions. Instead of taking a predefined set of metrics as a starting point, support was created for participants to add their own performance evaluation criteria.

The tool that was developed for supporting the service composition provides support during the five 
steps discussed in the previous subsection. Each stakeholder can add services, provide functional and non-functional descriptions for the services. The ability to select services and create a new composition and the function to evaluate the resulting compositions based on metrics like cost, reliability, etc. Often information will not be complete and the tool will only show what is known. Next, the participants can add their own expectations about the performance of services or use this to discuss the assumptions with others. In this way the tool is aimed at facilitating the steps and at supporting the identification of differences between starting points and objectives among stakeholders. In addition they can discuss their own preferred service compositions with each other. In this way it supports the creation of a dialogue among stakeholders, which should ultimately help to identify service compositions that are acceptable to all stakeholders.

\section{Results of comparing the methods}

The quasi-experimentation was evaluated by comparing the service composition method as currently used by the participants, labeled as 'best practice method' and the method proposed in this paper. We describe the quantitative and qualitative results in the following subsections.

\subsection{Quantitative results}

Our composition method relies heavily on group interactions and supporting the interactions among the stakeholders and the overcoming of the problems mentioned in section 2. In this light, the questions shown in table 1 were formulated to perform the quantitative evaluation. The scores of the best practice method and our method were compared using a paired t-test to determine if our methods was significantly better than the best practice method. The column named 'sig.' indicates if there is a significant difference in the scores between these methods. In total, 10 of the 18 questions show significant differences in favor of our method (given a $95 \%$ level of confidence). Three questions were even significantly different given a $99 \%$ confidence interval.

Table 1: Quantitative comparison of composition methods

\begin{tabular}{|c|c|c|c|c|c|c|}
\hline \multirow{2}{*}{$\begin{array}{l}N=21 \\
\text { Related questions: }\end{array}$} & \multicolumn{2}{|c|}{ Best Practice } & \multicolumn{2}{|c|}{ Multi Actor } & \multirow[b]{2}{*}{ Sig. } & \multirow[b]{2}{*}{ Z score } \\
\hline & Avg. & ttd. & Avg. & Std. & & \\
\hline 1 The support of functional requirements & 4,95 & 1,60 & 4,76 & 1,64 & & $-0,595$ \\
\hline 2 Managing a personal list of requirements & 2,90 & 1,67 & 4,57 & 2,01 & $* *$ & $-2,812$ \\
\hline 3 Making a requirements inventory with a group of involved parties & 4,05 & 1,66 & 5,24 & 1,30 & * & $-2,115$ \\
\hline 4 Support for requirement negotiation & 3,90 & 1,18 & 4,81 & 1,86 & * & $-2,156$ \\
\hline 5 Functional attributes are supported during evaluation & 3,81 & 1,54 & 4,57 & 1,69 & & $-1,867$ \\
\hline 6 Non-functional attributes are supported during evaluation & 3,05 & 1,60 & 4,19 & 2,32 & * & $-2,246$ \\
\hline 7 The composition method supports a set of evaluation methods & 3,14 & 1,49 & 3,76 & 2,32 & & $-1,179$ \\
\hline 8 The evaluation method supports attributes beyond functional fit & 3,38 & 1,60 & 4,90 & 1,67 & * & $-2,258$ \\
\hline 9 Costs, reliability and speed are evaluated & 4,67 & 1,28 & 5,57 & 1,25 & * & $-2,406$ \\
\hline 10 The composition method supports the creation of a shared view & 4,29 & 1,85 & 5,24 & 1,55 & $*$ & $-2,088$ \\
\hline 11 The shared view provides insight into the requirements & 4,05 & 1,69 & 4,52 & 1,86 & & $-1,100$ \\
\hline 12 The shared view provides insight into the services available & 4,24 & 1,55 & 4,67 & 1,88 & & $-0,915$ \\
\hline 13 The shared view enables communication between stakeholders & 4,00 & 1,92 & 5,19 & 1,47 & * & $-2,242$ \\
\hline 14 The service register also contains new services in short time & 2,90 & 2,10 & 2,81 & 2,29 & & $-0,248$ \\
\hline 15 It is possible to add new services to the register & 2,90 & 2,21 & 2,62 & 2,11 & & $-0,402$ \\
\hline 16 The register contains non-functional attributes of services & 2,24 & 1,51 & 4,38 & 2,38 & ** & $-3,074$ \\
\hline 17 The register provides evaluations of services by users & 2,52 & 1,75 & 3,86 & 2,24 & $* *$ & $-2,629$ \\
\hline 18 The register provides functionality to add/edit service attributes & 3,52 & 2,18 & 3,05 & 2,13 & & $-0,984$ \\
\hline
\end{tabular}

The participants were especially positive by the ability to manage personal requirements, to add and evaluate non-functional properties and to evaluate compositions. These aspects are related to the involvement of different stakeholders who might have different preferences. By including non-functional requirements, they were able to evaluate the effect on metrics like cost, quality, reliability, scalability and so on. They were able to add the type of performance indicators they preferred.

The evaluation shows that the participants found that the ability to add their own perspective was a benefit. They were able to make a set of requirements on the composition from their own point of view and share this with the other participants in the workshop. 
In this way support for requirement negotiation was created.

Also the adding of non-functional requirements was viewed as benefit of the composition method over the methods they used in their daily practice. In this way the cost, reliability of resulting composition and response time of a service can be evaluated. Response time was viewed as an important factor, as the subsequent invocation of web services causes overhead resulting in longer response time for the composition.

Further, the participants considered they way in which a shared view was created as better. The method and supporting tool show the services used in the compositions, the performance assumption underlying each of the services and this makes the desires of participants explicit. In this way the various stakeholder views were made visible to the participants.

Not surprisingly, the participants did not find that the method supported better functional requirements and the evaluation of functional requirements, as in their own practice the functions are also taking as a starting point. The participants indicated that the use of multiple evaluation methods was not viewed as a benefit of the supporting tool. In their own practices they were used to use various evaluation methods. Surprisingly the question results indicated that the shared view provided no more insight in the requirements and services available. The motivation of the participants was that an overview of services was always a necessary condition and that in one way or another a requirement would be discussed in the process. Also the ability to add services that are not developed yet was not considered as a difference with their own practice. They argued that they often discussed the development of new services, as the set of existing services is hardly never been able to create the desired new system or at least needs to be modified.

\subsection{Qualitative results}

Table 2 contains the strengths and weaknesses of the methods derived from observations and the comments made by the participants during the session and interviews. These qualitative results confirm the outcomes of the quantitative evaluation presented in table 1. The participants liked that the problem was taken as a starting point and that there were various ways to compare and discuss composition. Although the focus of the composition method is on dialogue and understanding each other interests and assumptions and avoiding judgmental pitfalls and advocacy of their own viewpoint, they felt that this cannot be avoided. They indicated that several times they felt that people were defending their position and judging others instead of looking for new solutions. They indicated that the role of the facilitator is crucial, as the facilitator should intervene in such situations and ensure that the rules of the game in the workshop are not violated.

Additional comments are related to the fact that the participants were familiar with the method used in their organizations, whereas they had no experience with the multi-actor composition method. The idea of bringing stakeholders together requires that they should be available at the same time, which was viewed as a weakness. The scheduling of such a session needs to be planned a long time in advance, as most stakeholders have a very busy schedule.

Our composition method does not generate a single outcome that is optimal in a certain way. It is aimed at supporting the creation of various service compositions and evaluating them. In this way trade-offs are made transparent and understandable to others. The participants indicated that his was also a weakness, as it is sometimes hard to understand which outcome would be the best.

Both the current methods and the multi-actor composition method are able to deal well with a set of services that are not interoperable and need to be modified to create a composition. The participants indicate that the reliance on experts was a strength but also a weakness at the same time. After understanding that formal methods can be used to prove the correctness, automatically generate result and so on, they would prefer such elements included in the method. In short, they would like to have the best of both worlds. As the starting points of the composition method and our multi-actor method are different, it might be difficult to combine both worlds and this needs further research. 
Table 2: Qualitative comparison of composition methods

\begin{tabular}{|c|c|c|}
\hline & Best practice method & Multi-actor Composition method \\
\hline & $\begin{array}{l}\text { - Proven method in other situations (for example based } \\
\text { on RUP, well known to the system developers) } \\
\text { - } \quad \text { Participants are familiar with the method in other } \\
\text { situations } \\
\text { - } \quad \text { Not formal and easy to work around certain aspect } \\
\text { - } \quad \text { Focus on creating working products } \\
\text { No detection of infeasible combinations of services }\end{array}$ & $\begin{array}{l}\text { - Takes the problem as a starting point and each } \\
\text { stakeholder can define their own requirements and } \\
\text { evaluation criteria for the compositions } \\
\text { - } \text { Enables comparison of multiple alternatives } \\
\text { - Facilitates comparisons in a group of actors and } \\
\text { makes requirements and desires on the composition } \\
\text { explicit } \\
\text { Focuses on dependencies among services and } \\
\text { feasibility of compositions } \\
\text { Enables the use of non-functional (QoS) data } \\
\text { regarding services } \\
\text { Process followed is easy to understand to non- } \\
\text { technical people ('this service register is comparable } \\
\text { to a shopping site where people compare different } \\
\text { articles and make recommendations') } \\
\text { Is able to deal with incomplete requirements }\end{array}$ \\
\hline 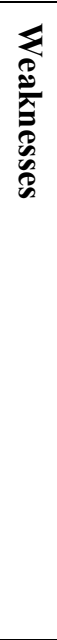 & 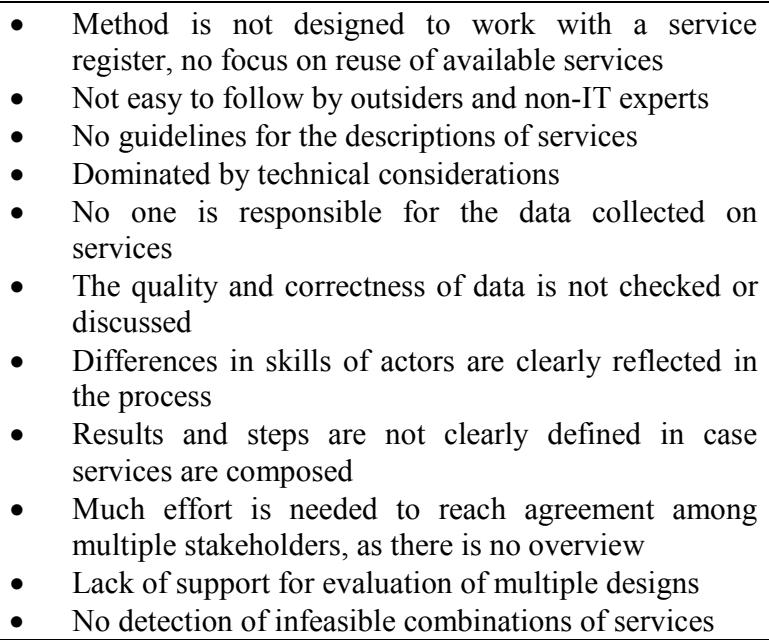 & $\begin{array}{l}\text { - No executable result, no formal correctness checks, } \\
\text { instead reliance of stakeholder judgment } \\
\text { No guaranteed outcome. Further, provides no single } \\
\text { outcome and participants are sometimes left puzzled, } \\
\text { there are many alterative having their own strengths } \\
\text { and weaknesses } \\
\text { - Demands involvement and time of all stakeholders } \\
\text { Depends on facilitator to keep to the rules of the } \\
\text { game. The starting point of the method that the focus } \\
\text { of the method is on exploiting rather than eliminating } \\
\text { each others viewpoints might not hold during a } \\
\text { workshop. Instead of having an open discussion, the } \\
\text { focus might shift on defending and attacking each } \\
\text { other viewpoints }\end{array}$ \\
\hline
\end{tabular}

\section{Conclusions}

Service composition might be viewed as a relatively straightforward process, but might be more complicated in reality. In this paper we bridged the gap between the creation of service compositions in pubic networks and existing service compositions methods. A multi-actor composition method was developed which is characterized by incomplete information, demand-driven approach, dealing with non-existing services, including non-functional requirements and the ability to deal with various stakeholder views. This resulted in a method which puts dialogue and conversational forms to the centre rather than optimization logic. The method is aimed at encouraging participants to think about the assumptions underlying their decisions and creating a shared view. In this way deeper inquiry into possible service compositions was created.
The method was evaluated using quasiexperimentation both quantitatively and qualitatively. This evaluation shows that the participants found that the ability to add their own perspective, manage their personal requirements, the inclusion of nonfunctional properties, the way compositions are evaluated are viewed as the main strengths. Furthermore, the participants liked that the problem was taken as a starting point and that various ways to compare and discuses compositions were provided. The evaluation showed that the focus on dialogues instead of judgment was sometimes difficult and depends on the skills of the facilitator.

The composition approach presented in this paper was primarily aimed at dealing with many stakeholders and on supporting group-wise learning, whereas other methods have relied on formal semantics. A logical next step would be to combine both approaches. The accomplishment of this combination is not easy as they might be conflicting 
in some sense, but might ultimately result in a more powerful composition approach.

The evaluations show that ways of handling the detection of infeasible combinations of services has not been given attention. Methods might generate combinations of services that are not feasible and therefore useless. We recommend that composition methods should include systematic analysis of the dependencies among services in compositions to ensure the generation of feasible combinations.

\section{References}

[1] M. Janssen, R. W. Wagenaar, and J. Beerens, "Towards a Flexible ICT-Architecture for MultiChannel E-Government Service Provisioning," presented at 36th Hawaii International Conference on System Sciences (HICSS-36), Big Island, Hawaii, 2003.

[2] W.-Y. Liang, C.-C. Huang, and H.-F. Chuang, "The design with object (DwO) approach to Web services composition," Computer Standards \& Interfaces, vol. 29, pp. 54-68, 2007.

[3] P. Fremantle, S. Weerawarana, and R. Khalaf, "Enterprise services. Examine the emerging files of web services and how it is intergraded into existing enterprise infrastructures," Communications of the $A C M$, vol. 45, pp. 77 - 82, 2002.

[4] F. Curbera, R. Khalaf, N. Mukhi, S. Tai, and S. Weerawarana, "The next step in Web Services," Communications of the ACM, vol. 46, pp. 29-34, 2003.

[5] R. W. Feenstra, M. Janssen, and R. W. Wagenaar, "Evaluating Web Service Composition Methods: The need for including Multi-Actor Elements," The Electronic Journal of e-Government, vol. 5, pp. 153-164, 2007.

[6] M. F. W. H. A. Janssen and A. S. J. R. Joha, "Motives for Establishing Shared Service Centers in Public Administration," International journal of information management, vol. 26, pp. 102 - 116, 2006.

[7] N. Milanovic and M. Malek, "Current solutions for Web service composition," IEEE Internet Computing, vol. 8, pp. 51 - 592004.

[8] D. L. Parnas, "On the criteria to be used in decomposing systems into modules," vol. 15, pp. 1053 - 1058, 1972.

[9] R. W. Feenstra, M. F. W. H. A. Janssen, and R. W. Wagenaar, "Designing a Service Composition
Framework to Support Multi Actor Networks," presented at EURO mGOV 2006, Brighton, UK, 2006.

[10] J. A. d. Bruijn and E. F. t. Heuvelhof, Process management: why project management fails in complex decision making processes. Dordrecht: Kluwer Academic, 2002

[11] H. B. Milward and K. G. Provan, "Managing Networks Effectively," presented at the 7th National Public Management Research Conference, Georgetown University, 2003.

[12] J. G. March and H. A. Simon, Organizations: John Wiley \& Sons Inc, 1958.

[13] H. A. Simon, The new science of Management Decisions. New York: Harper \& Row, 1960.

[14] J. A. d. Bruin and E. F. t. Heuvelhof, Netwerkmanagement, Strategieën, Instrumenten en Normen. Utrecht: Lemma, 1995.

[15] J. A. d. Bruin, E. F. t. Heuvelhof, and R. J. i. t. Veld, Procesmanagement, over procesontwerp en besluitvorming. Amsterdam: Academic Service, 1998.

[16] A. P. Sage and J. E. Armstrong, Introduction to Systems Engineering: Wiley, 2000.

[17] M. H. t. Beek, A. Bucchiarone, and S. Gnesi, $A$ Survey on Service Composition Approaches: From Industrial Standards to Formal Methods: Technical Report 2006-TR-15, ISTI, Consiglio Nazionale delle Ricerche, 2006.

[18] T. D. Cook and D. T. Campbell, QuasiExperimentation: Design and Analysis Issues for Field Settings: Houghton Mifflin Company, 1979.

[19] J. P. Walsh, "Managerial and organizational cognition: notes from at rip down memory lane," Organization science, vol. 6, pp. 280-321, 1995

[20] M. Burstein, C. Bussler, T. Finin, M. N. Huhns, M. Paolucci, A. P. Sheth, S. Williams, and M. Zaremba, "A Semantic Web Services Architecture," IEEE Internet Computing, vol. 9, pp. 72 - 81, 2005.

[21] E. H. Schein, "Kurt Lewin's Change Theory in the Field and in the Classroom: Notes Towards a Model of Managed Learning " Reflections: The SoL Journal, vol. 1, pp. 59-74, 1996.

[22] M. Jefferey and I. Leliveld, "Best Practices in IT Portfolio Management," MIT Sloan Management Review, vol. 45, pp. 41-49, 2004. 